\title{
Analysis of Rotorcraft Pilot-Induced Oscillations Triggered by Active Inceptor Failures
}

\author{
Michael Jones* \\ German Aerospace Center (DLR), Institute of Flight Systems, Braunschweig, Germany \\ Miles Barnett ${ }^{\dagger}$ \\ German Aerospace Center (DLR), Flight Experiments, Braunschweig, Germany
}

The use of active inceptor systems allows for control of the aircraft even during mechanical failures within the control inceptor. For the specific case of isometric failure, whereby the inceptor 'freezes' in position, a virtual force-displacement model is used to continue to provide control input. Experimental flight tests using the German Aerospace Center's (DLR) research helicopter (ACT/FHS) has shown the potential to encounter pilot-induced oscillation (PIO) tendencies when flying using this mode. This paper presents results from a simulation campaign undertaken to confirm whether PIOs may be exposed through this use of control and/or the resultant severity. The results show that the isometric failure mode caused severe PIOs. Unacceptable failure characteristics were reported by all four pilots. The use of offline prediction tools showed the incipience of PIO. It is recommended that specific PIO investigations are undertaken during the evaluation of active inceptor failure modes.

\section{Nomenclature}

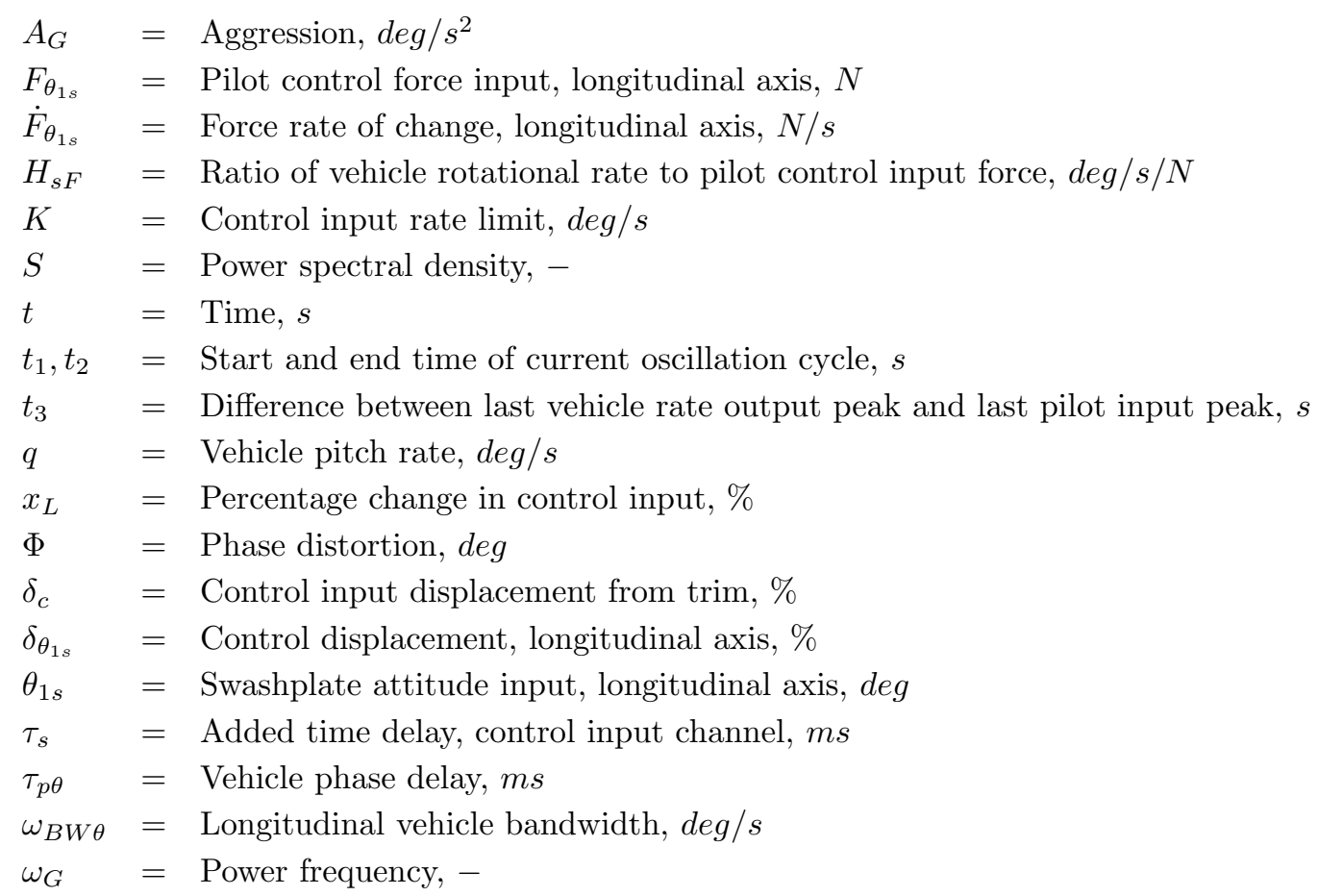

*Research Engineer, Rotorcraft Department, Lilienthalplatz 7, 38108 Braunschweig, Germany, michael.jones@dlr.de

†Experimental Test Pilot, Flight Experiments, Lilienthalplatz 7, 38108 Braunschweig, Germany, miles.barnett@dlr.de 


$$
\begin{aligned}
& \omega_{c o}=\text { Time-varying cutoff frequency, } \mathrm{deg} / \mathrm{s} \\
& \omega_{o} \quad=\text { Open-loop onset point, } \mathrm{deg} / \mathrm{s}
\end{aligned}
$$

$\begin{array}{ll}\text { ACT/FHS } & \text { Active Control Technology / Flying Helicopter Simulator } \\ \text { ADS } & \text { Aeronautical Design Standard } \\ \text { AIS } & \text { Active Inceptor System } \\ \text { A/RPC } & \text { Aircraft/Rotorcraft Pilot Coupling } \\ \text { APCR } & \text { Adverse Pilot Coupling Rating } \\ \text { AVES } & \text { Air Vehicle Simulator } \\ \text { CPU } & \text { Central Processing Unit } \\ \text { DLR } & \text { German Aerospace Center (Deutsches Zentrum für Luft- und Raumfahrt) } \\ \text { EP } & \text { Experimental Pilot } \\ \text { FCC } & \text { Flight Control Computer } \\ \text { FCS } & \text { Flight Control System } \\ \text { FCMC } & \text { Flight Control Mechanical Characteristics } \\ \text { FTE } & \text { Flight Test Engineer } \\ \text { HQ(R) } & \text { Handling Qualities (Ratings) } \\ \text { HQL } & \text { Handling Qualities Level } \\ \text { IFES } & \text { Integrated Failure Evaluation Scheme } \\ \text { MTE } & \text { Mission Task Element } \\ \text { OLOP } & \text { Open-Loop Onset Point } \\ \text { PAC } & \text { Phase-Aggression Criteria } \\ \text { PAO } & \text { Pilot-Assisted Oscillations } \\ \text { PF } & \text { Power Frequency } \\ \text { PIO } & \text { Pilot-Induced Oscillation } \\ \text { PIOR } & \text { Pilot-Induced Oscillation Rating } \\ \text { ROVER } & \text { Real-Time Oscillation Verifier } \\ \text { SAS } & \text { Stability Augmentation System } \\ \text { SP } & \text { Safety Pilot } \\ & \end{array}$

\section{Introduction}

The failure of mechanical control inceptors may result in catastrophic failure, through loss of control. ADS- $33^{1}$ defines catastrophic failure as a situation that prevents continued safe flight and landing. The probability per flight hour of such an occurrence must be less than $10^{-9} .^{1}$ The use of active inceptor systems (AIS) allows for the control of the vehicle even during mechanical failure of the control inceptor. Both position and force information are used by AIS to configure the flight control mechanical characteristics (FCMC). In the case of a failure to either the force or position system, the remaining functioning system can be used to provide input to the flight control computer (FCC). This system is known to be used in current aircraft. $^{2}$ The extent of testing of these modes and whether they have been used in-flight is not known. Whilst the system's redundancy should prevent loss of control, the probability of failure in AIS is predicted to be higher than equivalent mechanically-linked systems. This is due to the added complexity.

In a typical AIS, pilot force signals (measured by force sensors) are transmitted to a central processing unit (CPU). Required inceptor deflection is calculated according to defined FCMC. The inceptor deflection is controlled by the motor drive electronics which incorporate both a position sensor feedback and an electric servo actuator and thereby generate the control deflection at the grip. Both position and force sensor signals are transmitted to the FCC and the flight control system (FCS) via a digital bus. It is usual for the control input position sensor signal to be used as control input to the FCC/FCS.

The inceptor control forces are generated by integrated servo actuators and thus can be modified in real 
time, such as to assist the pilot though active haptic feedback. ${ }^{3}$ It has also been demonstrated in various studies that the handling qualities (HQs) of a helicopter may be optimized using AIS through adaptation of their FCMC to the aircraft dynamics. ${ }^{4}$ Until recently, AIS have been limited to technology demonstrators and experimental aircraft. However a number of new rotorcraft programs, such as the Bell-525 5 and CH$53 \mathrm{~K},{ }^{2}$ use AIS as the primary method of control.

In the case that the force sensor fails, movement of the control inceptor is still possible, although the pilot will feel no force feedback from the system. This case is termed "isotonic" (= constant force) failure. In the case that the servo-actuator fails, the position of the inceptor cannot be changed. However, in this case, the force sensor can be used to provide a pilot commanded input. This case is termed "isometric" (= constant position) failure and is the focus of this current study. In this case, the force replaces the position as input to the FCC/FCS. Within the FCC/FCS, a 'virtual' position is calculated based on the FCMC characteristics prior to isometric failure.

To prove that the 'back-up' mode of the AIS system prevents catastrophic failure, the HQs of the system must be investigated. In 2013, exploratory flight trials were undertaken in the Active Control Technology/Flying Helicopter Simulator (ACT/FHS), ${ }^{6}$ as the first steps to identify degradation in HQs during AIS failure. During the flight tests, the isometric failure case was found to expose pilot-induced oscillation (PIO) tendencies. The pilot commented that considerable pilot effort and attention was required to suppress oscillations. This was achieved through a looser grip of the inceptor.

At the time, a number of test cases were flown in a ground based simulation facility. Undertaking tests in simulation allowed for more dynamic manoeuvring and comments confirmed that PIO tendencies were prevalent in the system. In particular, the isometric failure case with extreme displacement was very difficult to control. Anatomical restrictions meant that the pilot was unable to reach the trim release button with the right thumb and so could not trim-out forces required to stabilise the aircraft. When trim forces could be removed, the control became much easier.

The flights in the simulator were found to be extremely fatiguing and hence it was subsequently more difficult to achieve consistently high accuracy. The pilot commented that following failure with extreme longitudinal displacement, recovery required approximately 1-2 minutes. This was to develop a new control strategy to suppress oscillations. This was only to maintain control of the vehicle and not to maintain some level of desired task performance. The pilot commented that the difficulties could be overcome after a period of practice, but that landing or performing additional tasks would likely still remain a challenge. In addition to comments regarding the longitudinal axis, failure during extreme lateral displacement also caused problems. However, this was easier to control and less fatiguing.

PIOs are a subset of aircraft/rotorcraft pilot couplings (A/RPCs). A/RPCs are adverse, unfavourable phenomena originating from undesirable couplings between the pilot and the vehicle. ${ }^{7,8}$ Typically, they are experienced during the development of novel control systems and during flight testing. PIOs are the most commonly cited A/RPCs, and the subset refers to oscillations which are the result of an active pilot within the control loop of the aircraft. PIOs can be classified into three groups. ${ }^{8}$ PIOs linear in nature, usually resulting from system time delays, are referred to as Cat. I. Quasi-linear behaviour, which may be caused by position or rate limits, are referred to as Cat. II type oscillations. Cat. III events are those where the vehicle response is non-linear. There is a crucial distinction between PIOs and other oscillatory vehicle responses. In the former case, the pilot is driven by the oscillations. In the latter, the pilot drives the oscillations. ${ }^{9}$ Typically PIOs occur between $1-10 \mathrm{rad} / \mathrm{s},{ }^{8}$ and pilot recognition is often the primary problem in their build-up. When possible, opening the control-loop can suppress oscillations. Oscillations where the pilot is passive within the control loop and where the oscillation frequency is greater than $10 \mathrm{rad} / \mathrm{s}$ are referred to as pilot-assisted oscillations (PAO). ${ }^{10}$ These can be prevented in a similar way. They are beyond the scope of this current paper. However, tools used here could be extended for future application to PAO problems. It is important to remember that any system can be driven to instability when forced. In this way, PIOs can always be exposed. However, it must be considered if the situation is appropriate and realistic.

$\mathrm{PIO} / \mathrm{PAO}$ phenomena have recently been cited as a factor in a number of fatal accidents occurring during flight testing. ${ }^{5,11}$ In Ref. 5, biodynamic feedback caused PAO phenomena in the Bell 525. In Ref. 11, a coupling between the pilot and the FCS of the AW609 caused divergent oscillations to occur following counter-phase pilot input. A/RPC phenomena are particularly dangerous due to the difficulty in defining the specific 'events' which cause them to occur. These are referred to as PIO trigger events, and are often first exposed only during flight test programs, and not during the pre-flight testing analysis.

This was the case found for both fatal accidents described above. As documented in the accident report 
of the AW609, ${ }^{11}$ two test runs under the same conditions and with the same aircraft configuration were flown prior to the fatal run. These were without incident. In the case of the accident, a more dynamic entry into the dive condition was made, which led to a consequential roll tracking sub-task. This unforeseen action (i.e. trigger situation) led to a divergent PIO.

AIS control failure has not previously been recognised as a PIO trigger. This was however found in the 2013 test campaign undertaken at DLR. Ref. 7 provides a detailed summary of emerging trigger situations. These include variable rotor speed, the influence of smart structures, and future vertical lift concepts. However, novel failure modes of AIS are not presented as possible PIO triggers. This suggests that there has not been enough consideration to date regarding the possibility of RPCs during this failure case.

The motivation of the current work is to determine why PIO tendencies were exposed during this case and provide an assessment of their hypothesised severity. Moreover, does the use of the AIS 'back-up' mode actually increase safety?

This paper presents the results from a study conducted in DLR's simulation facility, the Air Vehicle Simulator $\left(\mathrm{AVES}^{12}\right)$. One mission task element (MTE) was used during isometric failure investigations. Analysis is shown through the use of RPC prediction and detection techniques.

The paper proceeds as follows. Firstly, the Phase Aggression Criteria (PAC), used for objective analysis of the data, is revisited. Secondly, an analysis of PIO tendencies in the vehicle model used in this investigation is conducted. Following, the piloted simulation campaign is outlined, and results found are presented and evaluated. RPCs are assessed in terms of both objective and subjective analysis criteria. Finally, conclusions are drawn from the work, and recommendations are presented.

\section{Overview of the Phase Aggression Criteria (PAC)}

The Phase Aggression Criteria (PAC) was developed as a method for real-time detection of RPCs. Its use is specifically targeted at PIO events. The conception of the criteria, testing and subsequent validation is outlined in Ref. 13. The method stemmed from other concepts for observation of RPCs, including Real-Time Oscillation VERifer (ROVER) proposed by Mitchell ${ }^{14}$ and the Pilot-Inceptor Workload theory proposed by Gray. ${ }^{15}$ The theoretical application of the real-time capable method is shown in Fig. 1. Aggression $\left(A_{G}\right)$ is a measure of how intensely the pilot is working. Higher aggression means faster and/or larger control inputs. In previous investigations, only inceptor position signals were available. In this study, due to the nature of the isometric failure and the availability of the signal, the pilot force input at the inceptor is used. This replaces terms for position with terms for force in the equation to calculate Aggression (presented in Ref. 13). $A_{G}$ is found by finding the pilot input rate during the last oscillation cycle, and is calculated for the pitch axis using Eqn. 1.

$$
A_{G}=H_{s F} \frac{1}{t_{2}-t_{1}} \int_{t_{1}}^{t_{2}}\left|\dot{F}_{\theta_{1 s}}(t)\right| d t
$$

Here, $H_{s F}$ is the ratio of vehicle rotational rate to pilot control input force, $t_{1}$ and $t_{2}$ are parameter observation times, and $\dot{F}_{\theta_{1 s}}$ is the control force input rate (in the pitch axis). As displayed in Fig. $2, t_{1}$ is the time of the beginning of the current oscillation cycle and $t_{2}$ is the time at the end of the oscillation cycle. The parameter $H_{s F}$ is used to normalise $A_{G}$ based upon the relationship between pilot input and vehicle rate. Using force input, it is calculated using Eqn. 2.

$$
H_{s F}=\frac{\Delta q}{\Delta F_{1 s}}=\frac{\Delta \delta_{\theta_{1 s}}}{\Delta F_{1 s}} \frac{\Delta \theta_{1 s}}{\Delta \delta_{\theta_{1 s}}} \frac{\Delta q}{\Delta \theta_{1 s}}
$$

The use of $H_{s F}$ means that the units of $A_{G}$ remain as $\mathrm{deg} / \mathrm{s}^{2}$. This is considered applicable to ratecommand type systems. The use of other control system types has previously not been investigated during PAC, and will form part of future research efforts. In this research $H_{s F}$ is approximated as a scalar. The parameter $H_{s F}$ was determined from the calculation of vehicle control (force) input to aircraft rate output (for rate command systems), as shown in Eqn. 2. Here, $q$ is the vehicle pitch rate, $\Delta \theta_{1 s}$ is the longitudinal swash-plate output and $\Delta \delta_{\theta_{1 s}}$ is the control input. $H_{s F}$ gives an approximation of the control system sensitivity and is approximated as a constant. Phase distortion $(\Phi)$ is calculated using Eqn. 3 . Here, $t_{3}$ is the time between control input and vehicle rate output oscillation peaks. $\Phi$ is expressed in degrees. 


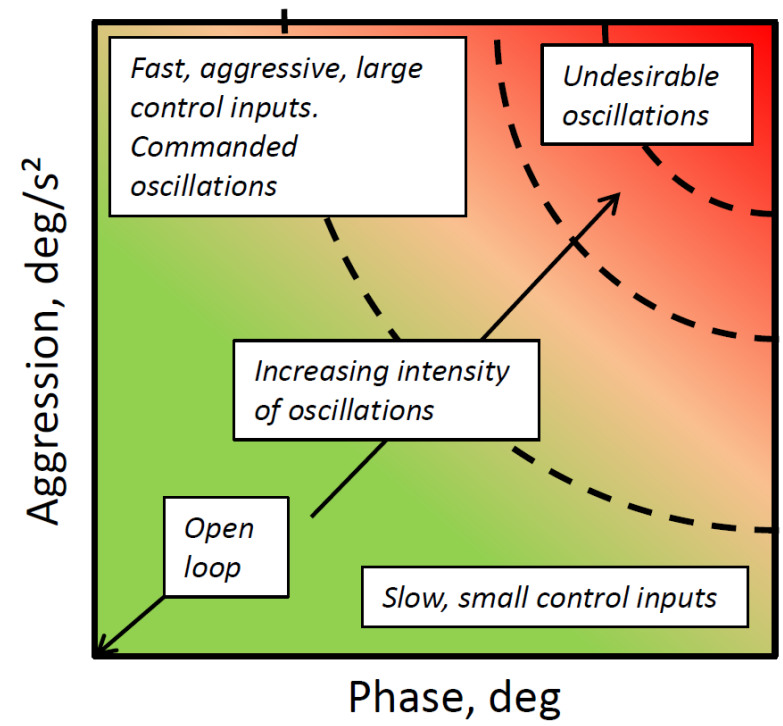

Figure 1: Schematic of Phase Aggression Criteria (PAC) detection algorithm

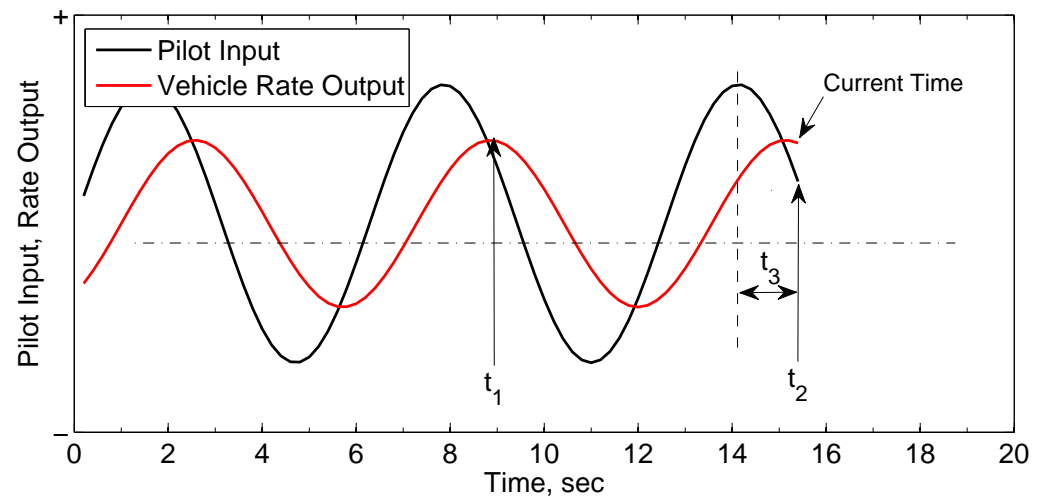

Figure 2: Calculation of PAC parameters

$$
\Phi=360 \frac{t_{3}}{t_{2}-t_{1}}
$$

If high $A_{G}$ is not accompanied by large $\Phi$ between input and output, oscillations are classified as commanded, driven by the pilot. Conversely, low $A_{G}$ denotes small or slow control inputs. In this case, high $\Phi$ between input and output may occur. However, this would not be a concern for PIOs. The case where high $A_{G}$ and high $\Phi$ are detected denotes PIO situations. This is where detection should occur.

When determining PAC results all control input and vehicle output signals are assumed to be oscillatory. Peaks and troughs in both input and output signals are compared in order to determine the $\Phi$. A distortion of $90^{\circ}$ between pilot input and vehicle output rate shows classical instability $\left(180^{\circ}\right.$ phase between pilot input and vehicle attitude output). Boundaries for the use of PAC were determined in previous research. ${ }^{13}$

Figure 3a displays a simulated case, whereby the crossover model, ${ }^{16}$ in conjunction with a simple tracking task and linear model of helicopter longitudinal dynamics have been used to generate a PIO case. The crossover model is used to tune the dynamics of the pilot model, to ensure correct behavior of the pilotvehicle system. In this case, the pilot crossover frequency remains constant throughout. To act as the trigger for PIO, a transport delay has been added at 20 seconds. This results in an increase in both vehicle rate and 


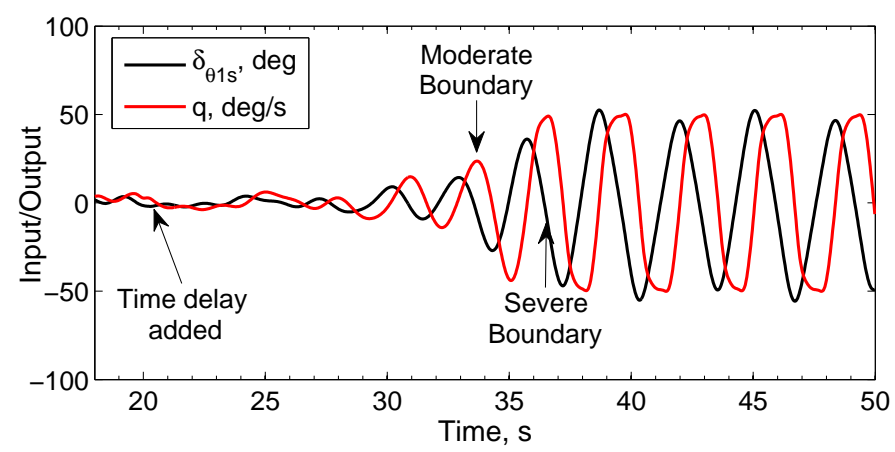

(a) Simulated data trace

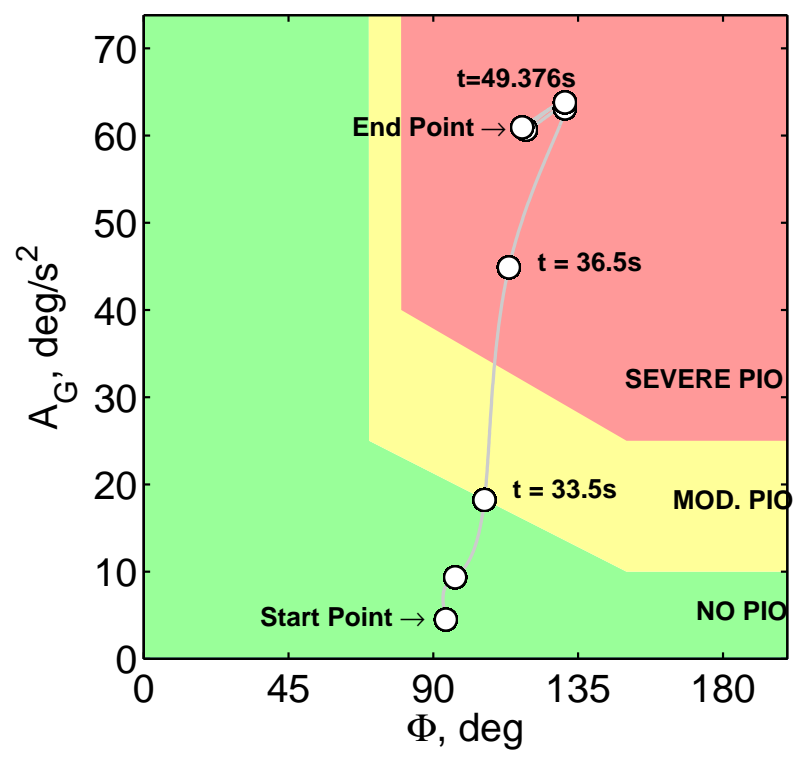

(b) PAC results shown with respect to longitudinal boundaries

Figure 3: Simulated data of a PIO case, driven through activation of additional transport delay.

pilot input magnitude. Actuator position limiting occurs after 35 seconds. These oscillations are sustained until the end of the simulation.

Figure 3b displays results obtained using PAC. Longitudinal boundaries are used to determine whether PIO has been observed. Prior to the addition of a time delay, the points are low aggression, within the 'No PIO' region. Following the additional delay, an increase in aggression occurs. The first point within the 'Moderate PIO' region occurs at 33.5 seconds. At this point, oscillations reach approximately $20 \mathrm{deg} / \mathrm{s}$. Points are detected in the 'Severe PIO' region at 36 seconds, where oscillation amplitude is greater than $40 \mathrm{deg} / \mathrm{s}$. In the seconds following, the aggression increases to a maximum of approximately $A_{G}=65 \mathrm{deg} / \mathrm{s}^{2}$.

A real-time implementation of the criteria developed using Matlab/Simulink ${ }^{\circledR}$ was used for the analysis. The time for both $A_{G}$ and $\Phi$ calculations were determined through observation in the time domain of peaks in the pilot input and vehicle output. Therefore, updated $A_{G}$ and $\Phi$ values were obtained every oscillation cycle.

The calculation of $\Phi$ in the time domain can be sensitive to noise. In order to filter this noise and focus on results causing concern for PIOs, two additional limits were used in the PAC calculation. PAC points were only calculated if;

- $F_{\theta_{1 s}}>4 \mathrm{~N}$. Force inputs below this were found to represent situations where the pilot applied low frequency, low amplitude, 'open-loop' type control inputs. Simulation was always initiated with trimmed 
control (i.e. $F_{\theta_{1 s}}=0$ )

- When the calculated frequency of the oscillation cycle was between 1-10 rad/s. This is the frequency range of concern for traditional PIOs.

Despite efforts, during data analysis PAC errors due to noisy phase calculations were occasionally observed and could not always be prevented. In order to generate a 'clean' plot of the PAC points, only points with a phase distortion less than $200 \mathrm{deg}$ were considered. Furthermore, if a single spurious PAC point was found in the moderate or severe regions, this case was denoted as 'No PIO', as any oscillations in these cases were not sustained. In future research, further refinements are recommended to ensure that the phase calculation is robust to noise in the system.

\section{Theoretical Appraisal of PIO Tendencies}

\section{A. Vehicle Model}

The ACT/FHS is an experimental research aircraft operated and maintained by DLR. It is a highly modified version of the EC135 helicopter. As a result, its characteristics in no way reflect those of the operational variant of the vehicle type. The experimental aircraft has been used to investigate AIS for many years. ${ }^{17}$

As the test campaign for this paper was conducted entirely in a ground-based simulation facility, the flight safety risks were completely mitigated. However, in order to apply a consistent approach to any subsequent campaigns in the ACT/FHS aircraft, the inherent safety concepts were considered and maintained throughout the simulator campaign. The ACT/FHS safety concept relies on the use of a 'firewalled' noncertified, flexible, experimental system (which includes the sidesticks, experimental displays, experimental FCC and the experimental test pilot (EP)). Outside of this firewall is the certified, quadruplex core system (which includes the core interface computer, conventional inceptors, smart actuators and the safety pilot (SP)). The main principle relies on the SP shadowing his conventional inceptors whilst the EP controls the aircraft. The SP is available to regain control of the aircraft from the EP within $400 \mathrm{~ms}$ in the event of a FCC failure, runaway, automatic monitor induced transfer or if the SP considers it necessary for safety of flight. Throughout manoeuvres flown using the experimental mode, the SP has direct view of the control input to the actuator through motion of his control inceptor. If the magnitude of control becomes extreme, control can be regained.

During this investigation, an experimental configuration featuring a rate command system was used. The FCS featured a basic 3-axis Stability Augmentation System (SAS) to improve the HQs of the bare airframe. The configuration was selected as it has been extensively tested in-flight and PIO tendencies have not been reported during normal operation. Further aspects regarding the HQs of this vehicle configuration are included in Ref. 18.

\section{B. Vehicle Rate and Runaway Limiters}

The ACT/FHS features actuator rate limiting in order to ensure hardware limits are not exceeded. In addition, to ensure that the SP can gain control within $400 \mathrm{~ms}$ following any unexpected failures or runaways in the EP control channel, the ACT/FHS experimental control system features variable limiting elements. These are referred to as 'runaway limiters'. Figure 4 shows the definitions of parameters used in the runaway limiters. These are implemented both for the cyclic and the collective control axes separately. Runaway limiters are described in detail in Ref. 19. During this investigation, a set of limiters previously used in flight investigations were used. These were representative of a safety case for low speed flying tasks. They were previously not found to cause PIOs during normal operation.

Limiters are characterised by two parameters, $x_{L}$ and $K$. Limiting is based upon control excursion from the last control input. Therefore, $x_{L}$ is the percentage change in control position, which is not necessarily the trimmed control position. The limiting is symmetrical.

Limiting is applied to the control input signal only when the change in actuator position is greater than parameter $x_{L}$. When the input is greater than this threshold value, the rate limit $K$ is applied. In previous investigations, parameters for runaway filters were determined by satisfying Eqn. 4 for operational manoeuvres undertaken using the ACT/FHS. 


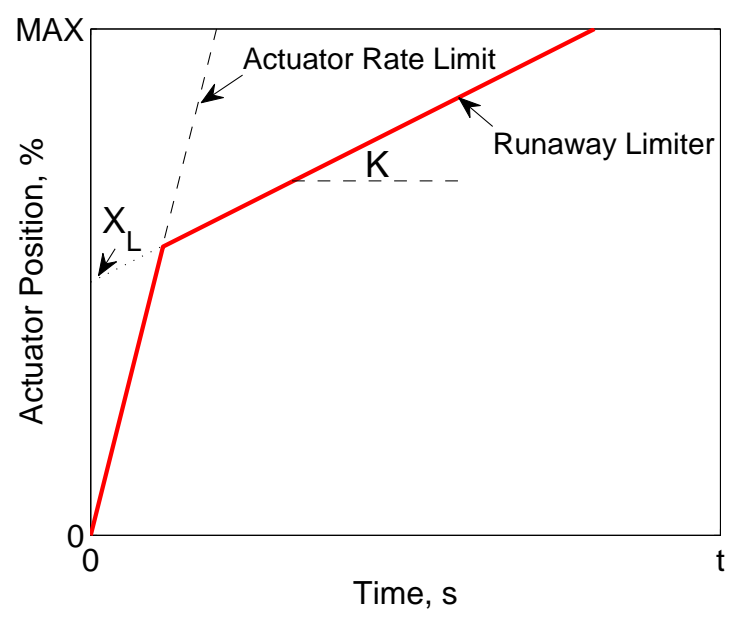

Figure 4: Definition of parameters in runaway filters

$$
\text { Control Rate } \leq K+x_{L} \frac{1}{\text { Input Duration }}
$$

\section{Vehicle HQs and RPC Triggers}

The PIO susceptibility of the vehicle was determined using both Cat. I (linear) and Cat. II (quasi-linear) prediction methods. The bandwidth phase delay criteria, as contained within ADS- $33^{1}$ gives an indication of Cat. I PIO susceptibility. This has been shown for both fixed- and rotary-winged vehicles, although differences exist in boundaries used. Studies detailed in Ref. 7 showed strong susceptibility to PIO with phase delay $\left(\tau_{p \theta}\right)$ greater than $200 \mathrm{~ms}$. This correlates well with ADS-33 lateral target tracking and acquisition boundaries but not with longitudinal HQ boundaries.

Figure 5 displays the longitudinal bandwidth determined for the control system used in this investigation with both no added time delay $\left(\tau_{s}=0 \mathrm{~ms}\right)$ and time delay $\left(\tau_{s}=200 \mathrm{~ms}\right)$. This was added in the forward control channel, between the pilot inceptor and the input to the control system. Results are shown for both force and position input.

In this case, the additional delay leads to a reduction in HQ level (HQL), from HQL 1 to HQL 2. Moreover, $\tau_{p \theta}$ increases to above $200 \mathrm{~ms}$. As given by the recommendations in Ref. 7 with $\tau_{s}=200 \mathrm{~ms}$, the configuration is predicted to be susceptible to Cat. I (linear) PIOs. For this reason, both the $0 \mathrm{~ms}$ and $200 \mathrm{~ms}$ cases were selected for investigation during the piloted simulation campaign.

For prediction of Cat. II type oscillations (quasi-linear), the Open-Loop Onset Point (OLOP) criteria was applied. $^{20}$ OLOP has been successfully used in many investigations. However, some disagreement with regards to the PIO susceptibility boundaries exist. Several studies have shown that PIO susceptibility boundaries are over-conservative for a variety of applications. ${ }^{21,22}$ Furthermore, a number of various methods to apply OLOP exist, such as whether (as originally specified) a pilot model is used and, if used, the fidelity of this model.

The OLOP criteria was applied to both actuator limiting only and runaway limiting cases. OLOP was applied using the method outlined in Ref. 23 using a pilot model tuned to give open-loop gain crossover $(0 \mathrm{~dB})$ at a phase of $-120 \mathrm{deg}$ (low pilot gain), shown in Fig. 6a and a phase of -160 deg (high pilot gain), shown in Fig. 6b. Based on previous research findings, results obtained from the application of OLOP with the high gain pilot model were expected to be conservative. ${ }^{24}$ PIO susceptibility based upon OLOP was determined using boundaries presented in Ref. 20. It has been suggested in previous work that the boundary for OLOP, using a pilot model, should be less conservative than the current OLOP boundary, with an amplitude of $2.5 \mathrm{~dB}$ between $-180 \mathrm{deg}$ and $-160 \mathrm{deg} .{ }^{13,21}$

To the authors' knowledge, this is the first application of OLOP to rate limiting elements dependent on control input magnitude. Therefore, it was necessary to make some considerations before its use. Using the 


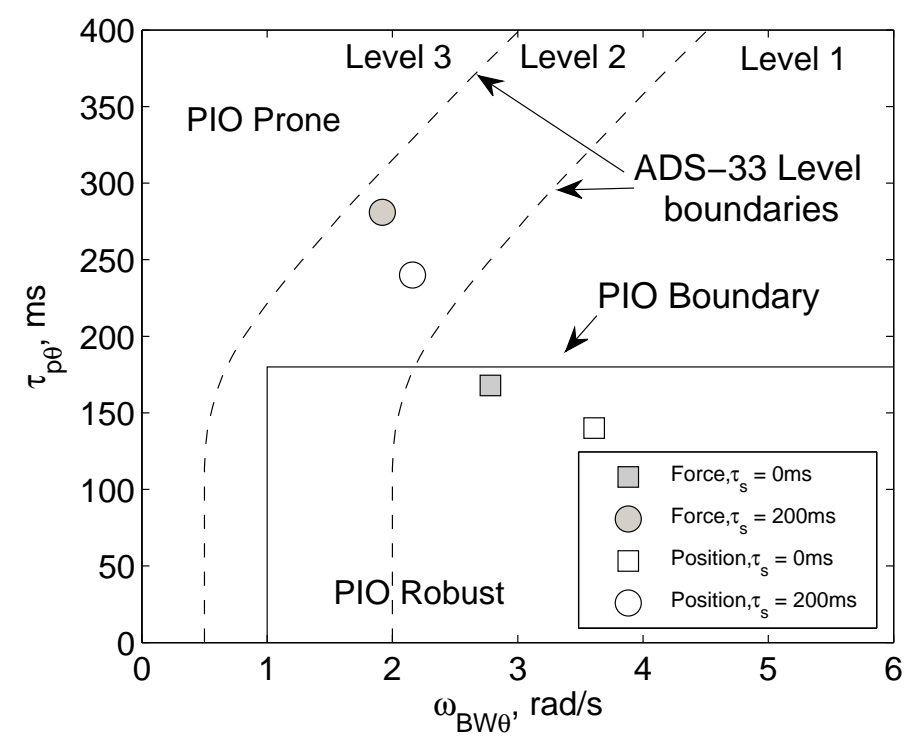

Figure 5: Bandwidth predictions for Cat. I PIO

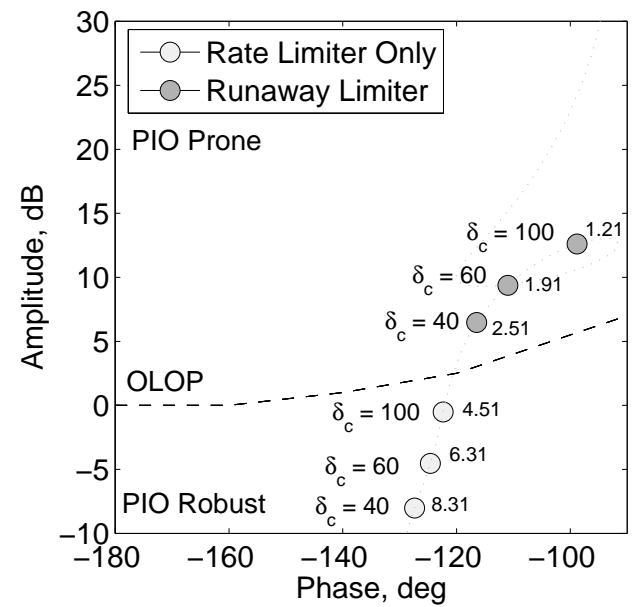

(a) OLOP predictions - with low gain pilot model

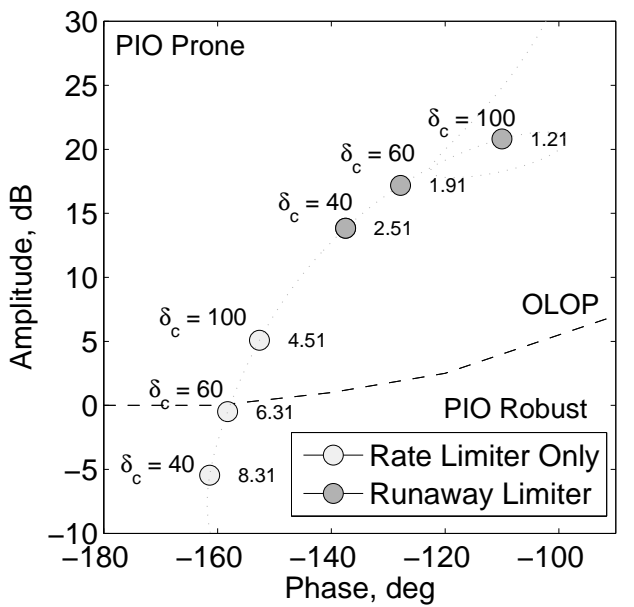

(b) OLOP predictions - with high gain pilot model

Figure 6: OLOP predictions

runaway limiters in this investigation, there is a region of actuator displacement that is not subject to rate limiting (from the runaway limiters). The approximation in this investigation is that this non-limited input is small during rate limiting. Therefore, runaway limiters are approximated as equivalent to a standard rate limiter. OLOP is usually calculated using maximum input amplitude $\left(100 \%\right.$ pilot input, $\left.\delta_{c}\right)$. In past research, this has led to conservative results. To fully investigate the results from OLOP, three control input magnitudes were investigated. Results are shown in Fig. 6.

Displayed are the pilot control input magnitudes ( $\delta_{c}$ in $\%$ of maximum travel) and the open-loop onset point $\left(\omega_{o}\right.$, displayed next to each point in $\left.\mathrm{rad} / \mathrm{s}\right)$. The onset point is the frequency at which the rate limiting elements will be 'activated'. Generally, the lower frequency of the onset point, the more likely that rate limits will be activated during flying tasks. Cat. II PIO is predicted for all control magnitudes investigated with the runaway limiter. For the rate limit only case, no PIO tendencies are predicted for the low gain pilot model. Using the high gain pilot model, Cat. II PIO is predicted for the case with maximum input 
amplitude but not for the $40 \%$ and $60 \%$ cases.

Addition application of OLOP following the method described in Ref. 24, without a pilot model resulted in no OLOP for the rate limit only configuration. Runaway limiter cases were found to be PIO prone, similar to results shown in Fig. 6. All results however were closer to the OLOP boundary than OLOP results using the pilot model.

In summary, results from the application of prediction methods indicate PIO susceptibility of the vehicle in the longitudinal axis. With the addition of $\tau_{s}=200 \mathrm{~ms}, \tau_{p \theta}$ increases above $200 \mathrm{~ms}$, which gives an indication of Cat. I PIO tendencies. With rate limiting only, Cat. II PIOs are only likely to be triggered with maximum control input and high pilot gain (i.e. the case used for OLOP analysis). This could perhaps occur in a failure situation. For the three limiting configurations, there is a strong indication that Cat. II PIOs may be triggered.

\section{Piloted Testing of PIO Tendencies}

The cases discussed in the preceding section were assessed in the AVES facility at DLR. Both subjective and objective assessment tools were used to analyse all data obtained from investigations conducted with four pilots. This section presents data obtained and analysis of important results. The correlation with objective tools is also discussed.

\section{A. Task Selection}

The MTE used in this study constituted a pseudo-random failure input during a continuous and repetitive series of manoeuvres at low speed and at low height. It was designed such that the pilot would only be required to fly the manoeuvre and, other than lookout, s/he would have no other tasks.

Figure 7 displays a schematic of the course used. Pilots were instructed by the flight test engineer (FTE) to fly to one of 8 azimuth directions (i.e. to either point A,B,C, or D). The pilot was required to perform a translation in the direction specified, achieving a groundspeed of 13-17 kts. During the task, the pilot is required to maintain groundspeed, heading and height $(15 \mathrm{ft})$. When reaching the hover point, the pilot is required to perform a smooth deceleration, with maximum decelerative attitude achieved immediately prior to the hover. This is followed by a stable hover satisfying tolerances in Table 1. During the deceleration, it was acceptable for the pilot to overshoot the target hover point slightly providing that $\mathrm{s} /$ he was still able to achieve the stabilised hover within the required time.

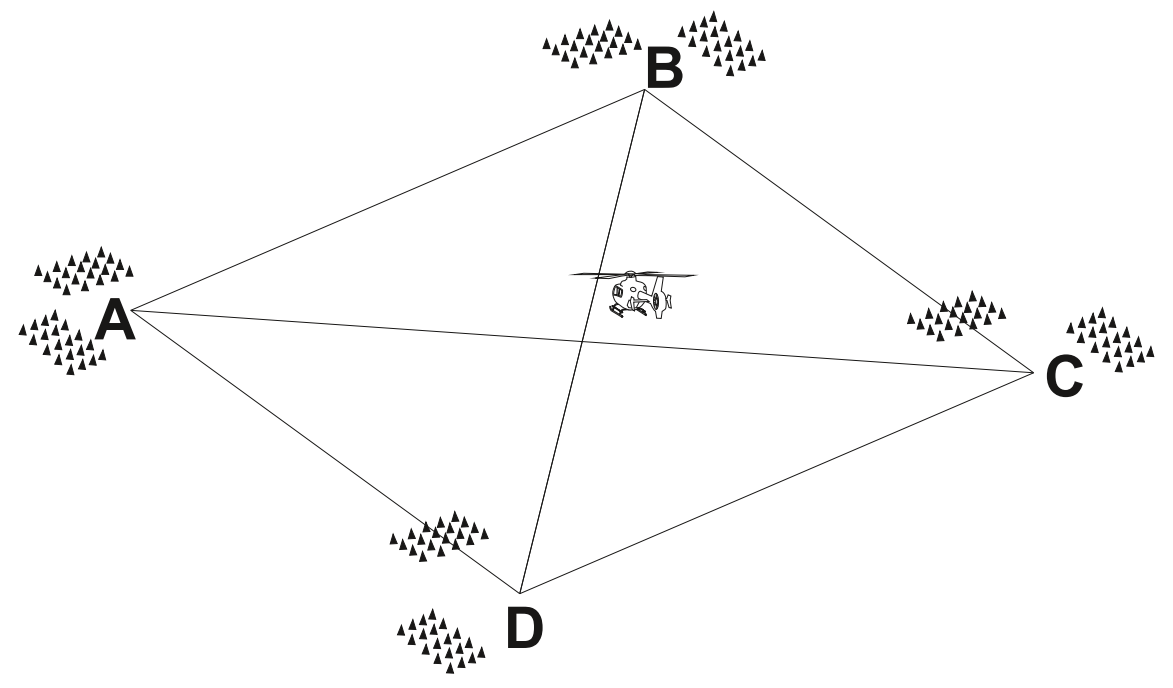

Figure 7: Schematic of test course

Multiple segments (i.e. each transition and hover pair) were completed sequentially. Following two to four successful segments, the AIS degradation to the isometric condition was triggered. This was unannounced, 
Table 1: Task performance requirements

\begin{tabular}{lcc}
\hline \hline Requirement & Desired Performance & Adequate/ Safe Recovery \\
\hline $\begin{array}{l}\text { Attain a stabilised hover within X seconds of initia- } \\
\text { tion of deceleration or failure, whichever comes latest }\end{array}$ & 6 & 10 \\
$\begin{array}{l}\text { Maintain a stabilised hover for at least X sec } \\
\text { Maintain the longitudinal and lateral position with }\end{array}$ & 20 & 20 \\
\pm X ft of a point on the ground & 3 & 6 \\
Maintain altitude within \pm X ft & 5 & 10 \\
Maintain heading within \pm X deg & 5 & 10 \\
There shall be no objectionable oscillations in any \\
$\begin{array}{l}\text { axis either during the transition to hover or the sta- } \\
\text { bilised hover }\end{array}$
\end{tabular}

and triggered manually by the FTE during the transition to hover, immediately before the pilot reached the stable condition. This was identified as the worst case failure situation during exploratory investigations as part of this research.

The high pilot feedback gain which was required to maintain the tight tolerances in the hover was designed to expose PIO tendencies. The pilot was instructed to prioritise task performance over PIO suppression. This was to simulate the scenario that the failure occurred during a period where the pilot must achieve hover in a confined area (e.g. landing in an urban environment). Neither the trim release nor the beeper trim functions were permitted during the MTE, due to comments made during the preliminary flight trials discussed. The test course shown in Fig. 7 was used as so the task could be reproduced in flight, without the need for significant fixed ground markers (moveable cones, markers). Four pilots were used in the investigation, their experience is shown in Table 2 .

Table 2: Experience of pilots used in investigation

\begin{tabular}{cccc}
\hline \hline \multicolumn{3}{c}{ Experience } \\
\hline Pilot & Flight Hours & Sim. Hours & Test Pilot \\
\hline A & 3000 & 400 & Yes \\
B & 1600 & 450 & Yes \\
C & 700 & 400 & No \\
D & 5400 & 900 & Yes \\
\hline \hline
\end{tabular}

\section{B. Qualitative Assessment}

Qualitative pilot comments were supported through the use of a number of subjective rating scales. To determine RPC/PIO incipience, two rating scales were used; the PIO rating scale and the Adverse Pilot Coupling (APC) scale. The original version of the PIO scale, presented in Ref. 25 and first used in 1967 (featuring only the use of descriptive terms) was used. This scale features no decision tree (added later in $\left.1981^{26}\right)$. In this work, ratings obtained using this scale are called 'PIOR'.

The PIOR scale is known to have a number of deficiencies, which lead to difficulties when classifying PIOs. ${ }^{27}$ One reason is the lack of guidance for pilots and the lack of consideration for non-oscillatory RPC phenomena (e.g. rapid divergence, loss of control). In Ref. 13, the APC scale was presented as an alternative means of assessing RPCs. This was developed through investigations during the European Union funded project ARISTOTEL (Aircraft and Rotorcraft Pilot Couplings: Tools and Techniques for Alleviation and Detection, No 266073, Ref. 7). The APC scale was developed with experimental test pilots, and detailed investigations comparing results obtained using previous scales were conducted. ${ }^{13}$ The scale is presented in Fig. 8. The layout has been refined from previous publication, to all the scale to be used on an in-flight kneeboard. Ratings obtained using this scale are termed 'APCR'. 
The Integrated Failure Evaluation Scheme (IFES) was used to determine the severity of the isometric failure. Initially developed by Hindson, Eshow and Schroeder in $1990,{ }^{28}$ IFES was designed to evaluate the effects of actuator hardover / runaways on the safety of flight and pilot recovery action for the variable stability UH-60 research helicopter.

The evaluation scheme is shown in Fig. 9. It is used to evaluate firstly the transient aircraft response and secondly the recovery handling. The IFES is a decision tree based scheme. The main question that it asks the test pilot is whether transients and the ability to recover from the failure were safe (tolerable) or unsafe (intolerable). Thereafter, the test pilot is required to assign separate ratings for the transient and recovery. The transient ratings were based upon the descriptions of aircraft state excursions and proximity to limits or obstructions and for the recovery rating they were based upon the urgency, effort and extent of the required corrective action. For the purposes of this study the scale was slightly modified from the original to include the effort required by the pilot in the recovery action for ratings B (low pilot effort) and C (moderate pilot effort). Also for the purposes of this study, 'Safe Recovery' was defined as maintaining the aircraft within the adequate performance tolerances and 'Loss of Control' was defined as the inability to land the aircraft on an unlimited flat surface.

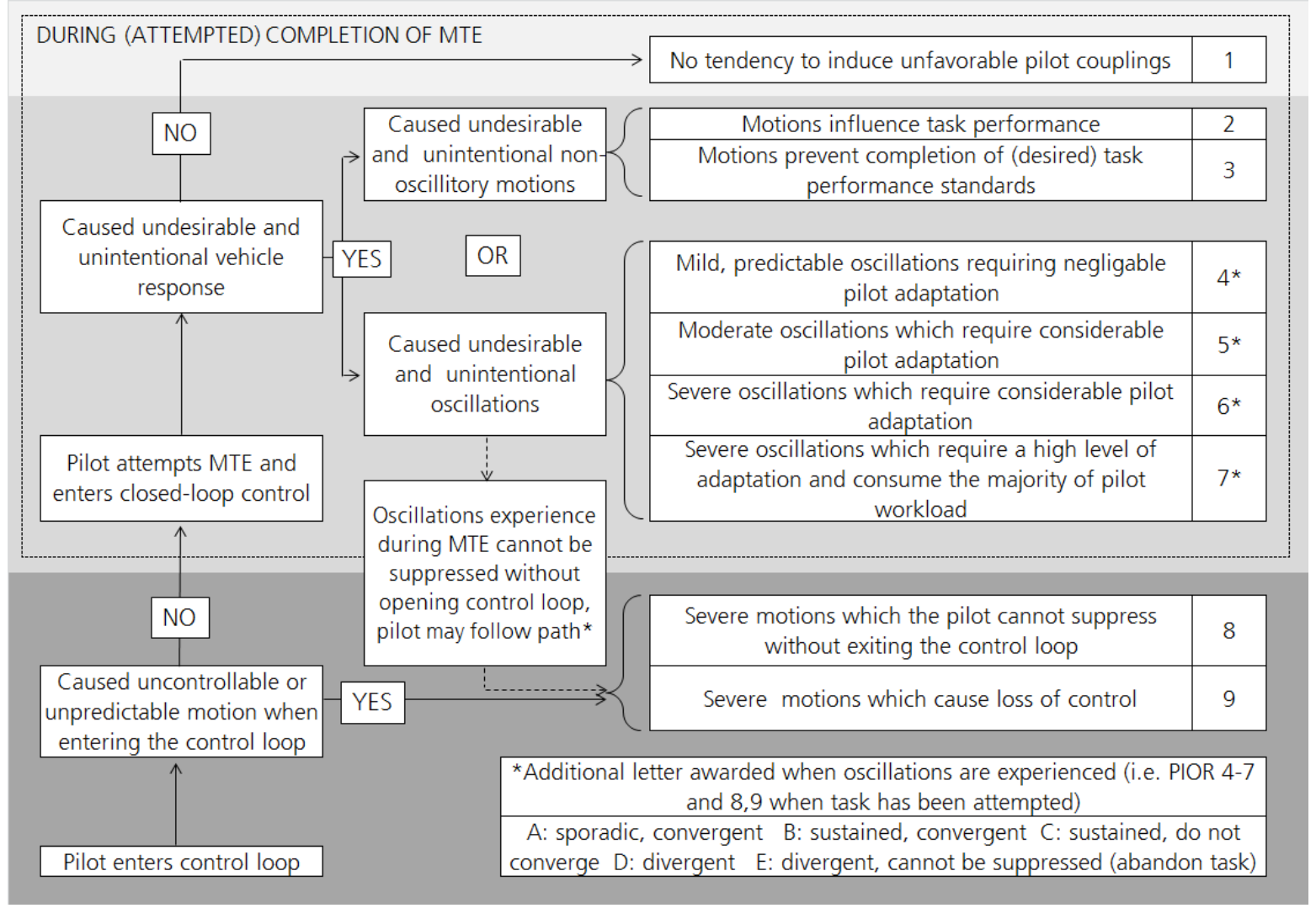

Figure 8: Adverse Pilot Coupling (APC) scale

\section{APCR/PIOR Ratings}

During the investigation, six test cases (Cases 1-6) were selected to give a range of PIO susceptibility. These cases are shown in Table 3. A combination of runaway limits, isometric failure, and time delays were used. Cases 1-3 were without isometric failure. Table 3 and Table 4 show APCR and PIOR ratings obtained for all test cases.

The overall results show that severe PIOs were frequently experienced following failure. However, this was in contrast to results obtained without failure. For at least one test point following isometric failure, each pilot was forced to abandon the task as a result of PIOs experienced. Many cases were awarded APCR $\geq 6$, denoting oscillations or non-oscillatory response which the pilot found severe. The PIOR scale cannot 


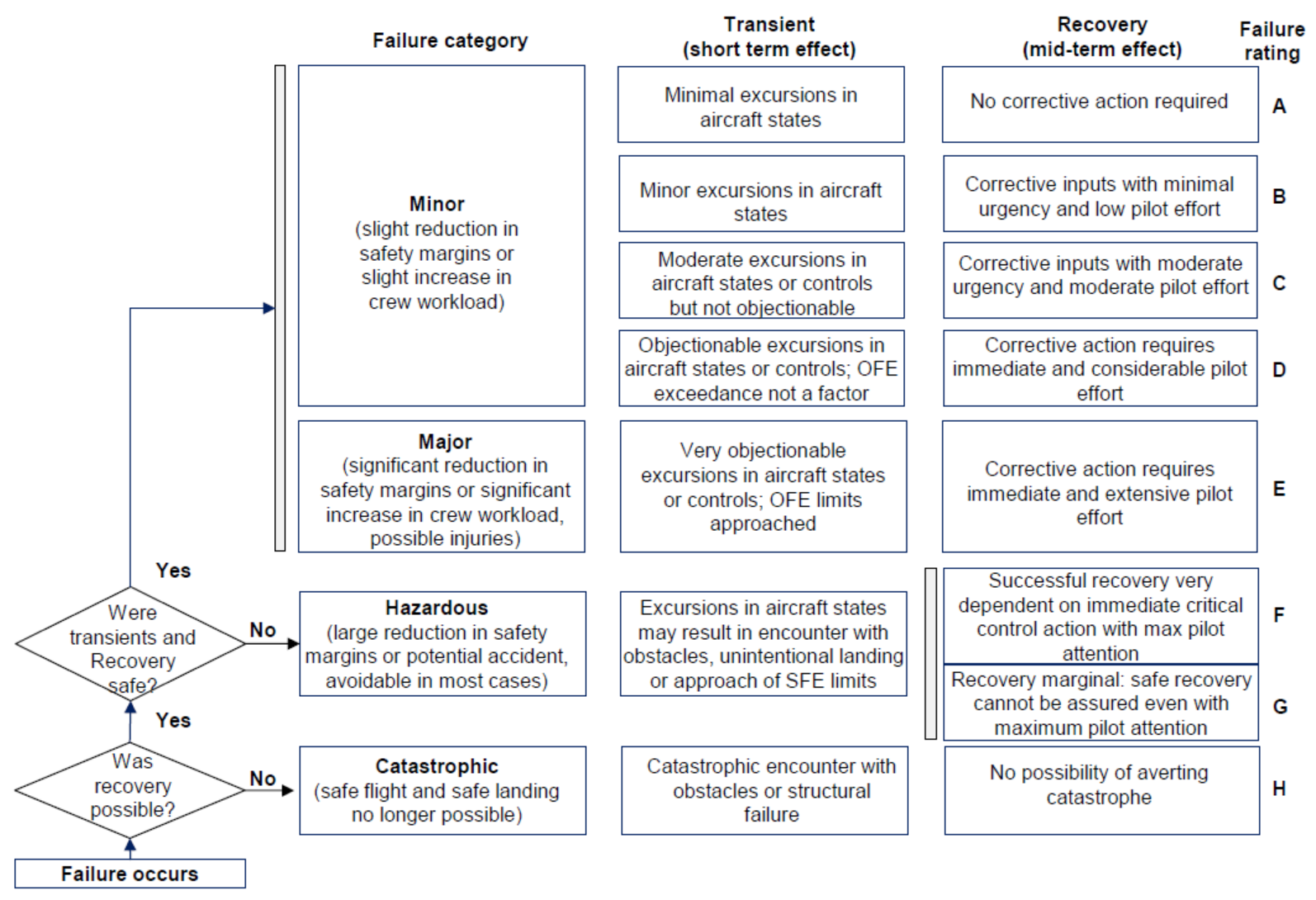

Figure 9: Integrated Failure Evaluation Scheme (IFES)

Table 3: APCR ratings obtained during isometric failure cases (number in brackets denotes number of occurrences)

\begin{tabular}{cccccccc}
\hline \hline & & \multicolumn{7}{c}{ Pilot } \\
\hline Case & Runaway Limits & Failure & Time Delay, ms & A & B & C & D \\
\hline 1 & No & No & 0 & 2 & 2 & - & - \\
2 & Yes & No & 0 & 1,2 & 1 & - & 3 \\
3 & Yes & No & 200 & 1 & - & - & 2 \\
\hline 4 & No & Yes & 0 & $7 \mathrm{D}(4)$ & $5 \mathrm{~B}, 6 \mathrm{D}$ & - & - \\
5 & Yes & Yes & 0 & $8 \mathrm{E}$ & $4 \mathrm{~B}, 6 \mathrm{D}, 7 \mathrm{D}, 7 \mathrm{E}, 8 \mathrm{E}$ & $5 \mathrm{~B}, 7 \mathrm{D}$ & $9(2)$ \\
6 & Yes & Yes & 200 & $8 \mathrm{E}(2)$ & $9 \mathrm{E}(3)$ & $9(2)$ & 8 \\
\hline \hline
\end{tabular}


Table 4: PIO ratings obtained during isometric failure cases (number in brackets denotes number of occurrences)

\begin{tabular}{cccccccc}
\hline \hline & & & \multicolumn{5}{c}{ Pilot } \\
\hline Case & Runaway Limits & Failure & Time Delay, ms & A & B & C & D \\
\hline 1 & No & No & 0 & 2 & 2 & - & - \\
2 & Yes & No & 0 & 1,2 & 1 & - & 1 \\
3 & Yes & No & 200 & 1 & - & - & 2 \\
\hline 4 & No & Yes & 0 & $3(2), 4(2)$ & 2,4 & - & - \\
5 & Yes & Yes & 0 & 6 & $3(2), 4(2), 6$ & 3,4 & $6(2)$ \\
6 & Yes & Yes & 200 & $5(2)$ & $4,6(2)$ & $6(2)$ & 5 \\
\hline \hline
\end{tabular}

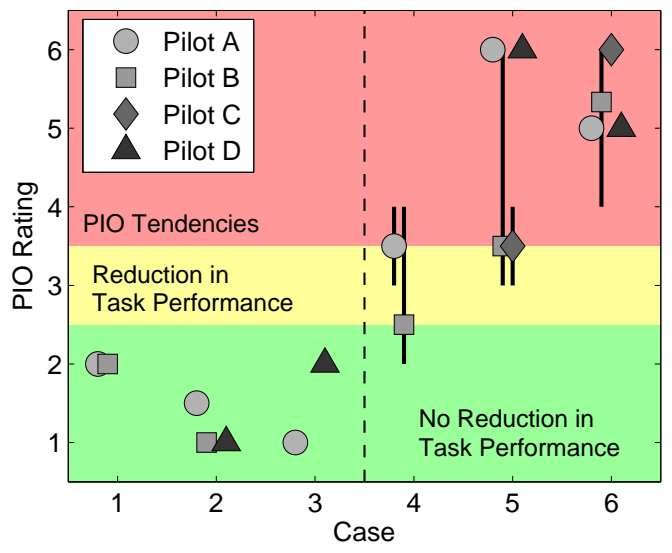

(a) PIORs awarded

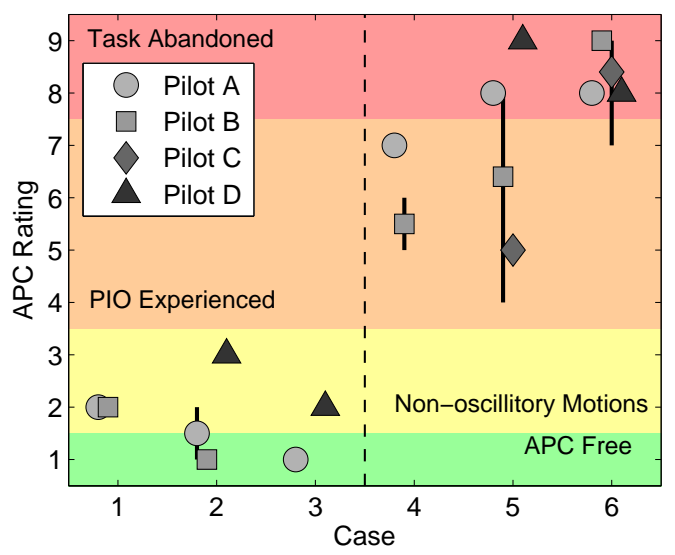

(b) APCRs awarded

Figure 10: Subjective APC/PIO ratings

be used to directly ascertain perceived severity of oscillations, but ratings PIOR $\geq 4$ usually denote that the oscillations had an impact on task performance. Ratings show that PIOR $\geq 4$ were consistently obtained throughout the investigation. Figure 10a displays PIO ratings awarded during the investigation. These are seperated with respect to assessing pilot and case number. All pilots experienced severe and divergent PIOs following failure with a $200 \mathrm{~ms}$ time delay. Intensity of oscillations generally decreased without the time delay. However the difference between failure and no-failure cases is clearly shown.

Figure 10b displays APC ratings awarded for the same test points. These ratings reflect PIOR. Both with and without time delay, Pilot D experienced non-oscillatory motions. Pilot D's task performance was also generally poorer than the other pilots. Moreover, the pilot was much more aggressive during completion of the MTE, therefore exposing more dynamics of the vehicle during the cases without failure. There was no clear difference found between cases with and without time delay without failure.

Results show a correlation between APCR and PIOR. Overall, APCRs are considered to give greater detail and understanding of pilot experiences during completion of the MTE. One reasons is that the ratings directly convey both the severity and nature of oscillations experienced. Information regarding severity are only indirectly obtained when using the PIOR scale. Strong correlation was found between APCR $=8-9$ and $\mathrm{PIOR}=6$. These ratings define situations where the pilot must either abandon the task or cannot enter the task. The majority of cases where $\mathrm{APCR}=4-5$ were found were awarded PIO $=3$, whilst the majority of cases where APCR $=6-7$ where found were awarded PIO $=4-5$.

The APCRs also directly show whether oscillations were considered convergent or divergent during the MTE. Whilst these terms are contained in the PIO scale, they are only included along with the complete description. As a result, PIOR $=4$ can denote convergent or divergent oscillations. It was also found that 
the characteristics of the oscillations (e.g. convergent, sustained, or divergent) were not always apparent from the use of the PIOR scale. As the original table version only was used (i.e. no decision tree), PIOR = 4 was found to denote both divergent and convergent oscillations.

It is important to mention that a major difference in PIO ratings may occur when using alternative rating scales (i.e. the 'combined scale' which is also frequently used). Despite the same numerical ratings, PIORs obtained using these two different scales should not be considered equivalent. Using the combined scale, PIOR $=5$ must denote divergent oscillations.

\section{IFES Ratings}

All IFES ratings obtained for isometric degradations that were initiated at the worst case time of failure are contained in Table 5. Using the form $\mathrm{X}-\mathrm{Y}(\mathrm{Z}), \mathrm{X}$ is the transient rating awarded, $\mathrm{Y}$ is the recovery rating, and $\mathrm{Z}$ is the number of ratings obtained.

Table 5: IFES ratings (numbers in brackets denote number of occurrences)

\begin{tabular}{ccccc}
\hline \hline \multicolumn{5}{c}{ Pilot } \\
\hline Case & A & B & C & D \\
\hline 4 & E-E,FG-E,FG-F,FG-G & D-C, C-D & - & - \\
5 & H-H & D-C, E-D, FG-F, FG-G, FG-H & FG-F & H-H $(2)$ \\
6 & FG-G(2) & H-H $(3)$ & H-H $(2)$ & H-H \\
\hline \hline
\end{tabular}

The worst IFES ratings obtained by each pilot are shown in Fig. 11. For one case, Pilot B awarded D-C and C-D IFES ratings. For this case, C-D is considered worse, and recovery is harder to achieve. The ratings show that, for Case 5 and Case 6, all pilots believed that the failure characteristics were unacceptable, both for transient and recovery phases of the flight. This assessment was both with and without added time delay. Three of the four pilots rated the case with added time delay $\mathrm{H}$ (transient) and $\mathrm{H}$ (recovery). This is the worst possible rating that may be obtained. For some of the cases, the failure characteristics deteriorate between transient and recovery phases. This strongly suggests that PIOs have been experienced. For Case 4, without runaway limiters, IFES ratings were more favorable. Only two of the pilots flew this case. Pilot $\mathrm{B}$ commented that the failure was acceptable, which reflects the data recorded.

For almost every test point completed with isometric failure, Pilot D either lost control or failed to prevent a catastrophic encounter. Furthermore, Pilot D was the only pilot who consistently could not attempt the MTE following the failure. For the majority of cases, all other pilots could attempt the MTE following failure, at which point severe oscillations were encountered.

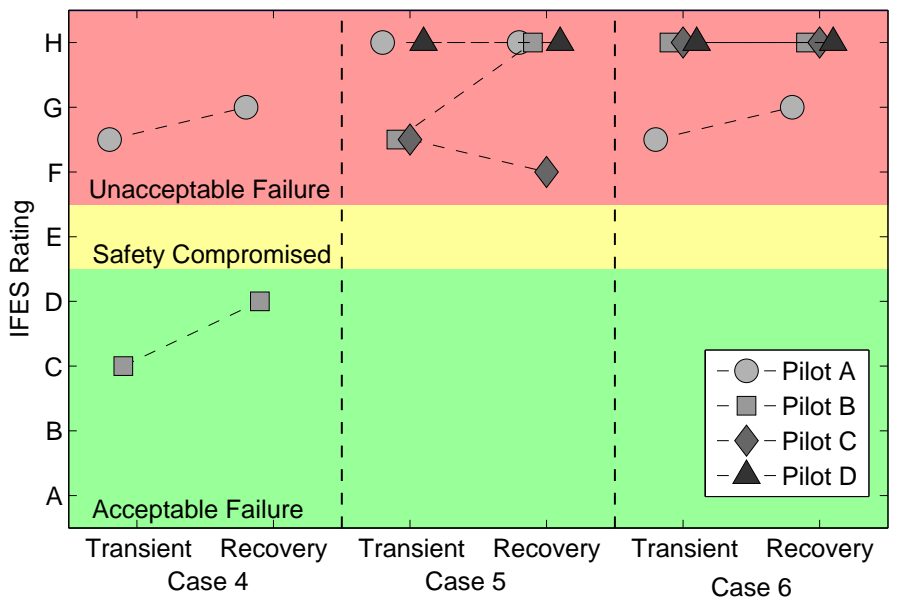

Figure 11: IFES worst case ratings obtained 


\section{E. PAC Criteria and Correlation}

A full analysis of all results obtained from the investigation was undertaken using PAC, with boundaries presented in Fig. 3b. As the focus of this investigation was the longitudinal axis, only this axis was investigated using PAC. During the flight testing and subsequent investigations in the simulator this case was found to expose greater PIO tendencies. Observations during piloted tests in this investigation confirmed this. Table 6 displays all PAC detections found with respect to case: No oscillations (N), Moderate oscillations (M), and Severe oscillations (S). Table 7 shows these detections with respect to APC rating awarded.

PAC was used to process all data recordings obtained during the investigations. Overall, PAC was found to correlate almost perfectly with pilot ratings. One case where rapid loss of control occurred (Pilot D) was not detected by PAC. This was as no oscillations were experienced, rather non-oscillitory departure. Rapid departure meant there were no full oscillation cycles and the algorithm could not calculate phase distortion. This is a limitation of PAC, whereby for rapid departures it may not provide information required. In this case however a dramatic change in $A_{G}$ was evident.

The majority of test cases flown were without failure. For these cases, PAC predominantly detected no oscillations (as expected). For a number of cases, moderate PIO was detected. In these cases, only a small number of PAC points were found within the moderate region. PAC detected either Moderate or Severe oscillations for almost all of the failure cases. The one exception was the rapid loss of control case discussed above. For all cases with failure and time delay, PAC detected severe oscillations.

Table 6: PAC observations (numbers in brackets denote number of occurrences)

\begin{tabular}{ccccc}
\hline \hline \multicolumn{5}{c}{ Pilot } \\
\hline Case & $\mathrm{A}$ & $\mathrm{B}$ & $\mathrm{C}$ & $\mathrm{D}$ \\
\hline 1 & $\mathrm{~N}(6), \mathrm{M}(3)$ & $\mathrm{N}(7), \mathrm{M}(1)$ & - & - \\
2 & $\mathrm{~N}(6)$ & $\mathrm{N}(20)$ & $\mathrm{N}(7)$ & $\mathrm{N}(10), \mathrm{M}(2)$ \\
3 & $\mathrm{~N}(8)$ & $\mathrm{N}(5), \mathrm{M}(1)$ & $\mathrm{N}(5)$ & $\mathrm{N}(2), \mathrm{M}(2)$ \\
4 & $\mathrm{~S}(4)$ & $\mathrm{S}(2)$ & - & - \\
5 & $\mathrm{~S}(2)$ & $\mathrm{M}(1), \mathrm{S}(3)$ & $\mathrm{M}(1), \mathrm{S}(2)$ & $\mathrm{N}(1)^{a}, \mathrm{M}(1), \mathrm{S}(1)$ \\
6 & $\mathrm{~S}(2)$ & $\mathrm{S}(3)$ & $\mathrm{S}(2)$ & $\mathrm{S}(2)$ \\
\hline \hline
\end{tabular}

${ }^{a}$ Case Resulted in Rapid Loss of Control

APC ratings reflect PAC results found. For Pilot B, two of the ratings did not correlate perfectly with PAC detections. Pilot B awarded 4B (minor oscillations) for one case where PAC detected severe oscillations. Furthermore, for a case where PAC detected moderate oscillations, Pilot B awarded 7D (severe divergent oscillations).

Table 7: APC ratings with respect to PAC detection (numbers in brackets denote number of occurrences)

\begin{tabular}{ccccc}
\hline \hline & \multicolumn{5}{c}{ Pilot } \\
\hline PAC Detect. & $\mathrm{A}$ & $\mathrm{B}$ & $\mathrm{C}$ & $\mathrm{D}$ \\
\hline $\mathrm{N}$ & 2 & 1,2 & - & $3,9^{a}$ \\
$\mathrm{M}$ & 2 & $2,7 \mathrm{D}$ & $5 \mathrm{~B}$ & 2 \\
$\mathrm{~S}$ & $8 \mathrm{E}(3), 7 \mathrm{D}(4)$ & $4 \mathrm{~B}, 5 \mathrm{~B}, 6 \mathrm{D}(2), 7 \mathrm{E}, 9 \mathrm{E}(3)$ & $7 \mathrm{D}, 9(2)$ & 8,9 \\
\hline \hline
\end{tabular}

${ }^{a}$ Case Resulted in Rapid Loss of Control

Despite a small number of inconsistencies, results further validate PAC boundaries developed during previous investigations. The single boundary appears suitable alone for RPC detection. As these results have been obtained in a new simulation environment, using a different aircraft model, PIO triggers, and pilots, the results further justify the use of the boundaries previously developed.

Figure 12 shows one example of the results of the PAC detection with respect to time. The case shown is for a severe PIO experienced by Pilot B whilst completing Case 6. The point at which position remains 
constant is the point that the isometric failure has occurred. Phase, Aggression, and frequency are calculated for each oscillation cycle. Each diamond represents a calculated point. As shown, for this case, the Aggression increases rapidly after isometric failure and begins to decay for the following 30 seconds. Following this decay however, the Aggression again begins to rise, a result of larger control inputs at similar frequencies. This case is shown with respect to PAC boundaries in Fig. 13f.

Figure. 13 displays an example of data recorded for each of the Cases. This shows the range of PAC results obtained during the investigations. Figures. 13a - 13c display results from cases where isometric failure did not occur. As shown, APCR and PIOR do not suggest PIOs to cause concern, which is reflected by the PAC chart. For cases where isometric failure occurs, Severe PIOs were found. Agreement is found between the APCR and the PAC detection.
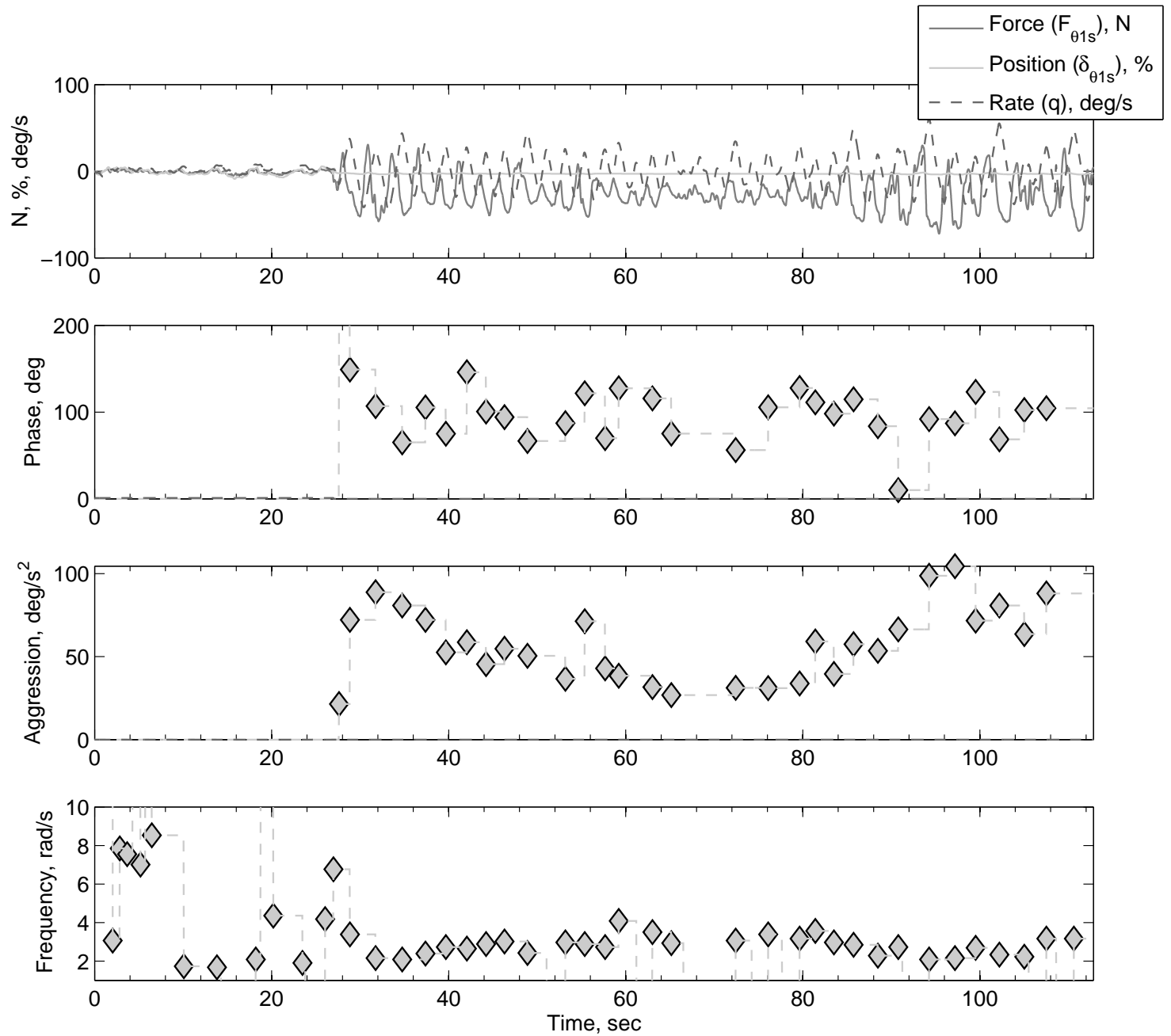

Figure 12: Example of PAC real-time detection algorithm output 


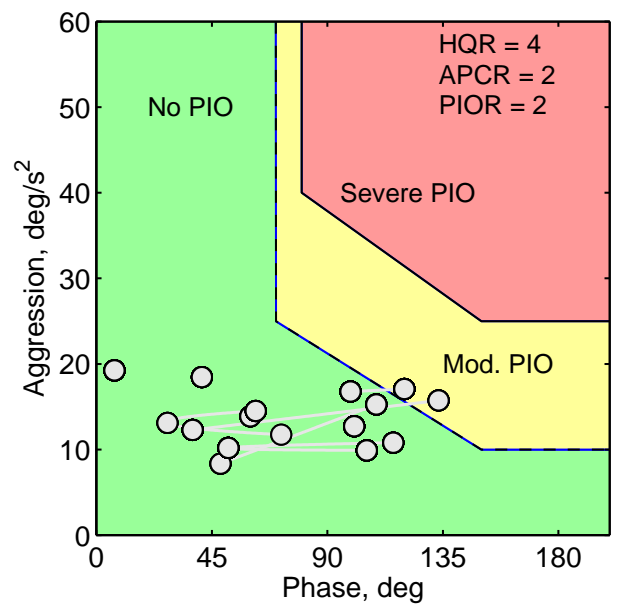

(a) PAC results - Case 1 - Pilot A

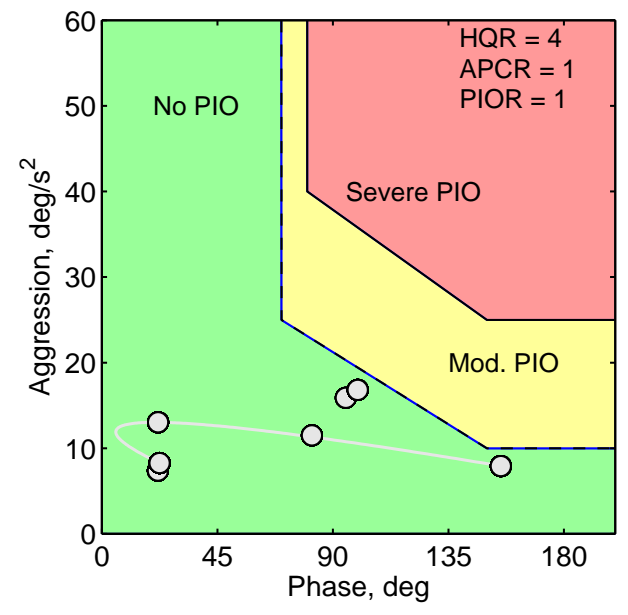

(c) PAC results - Case 3 - Pilot A

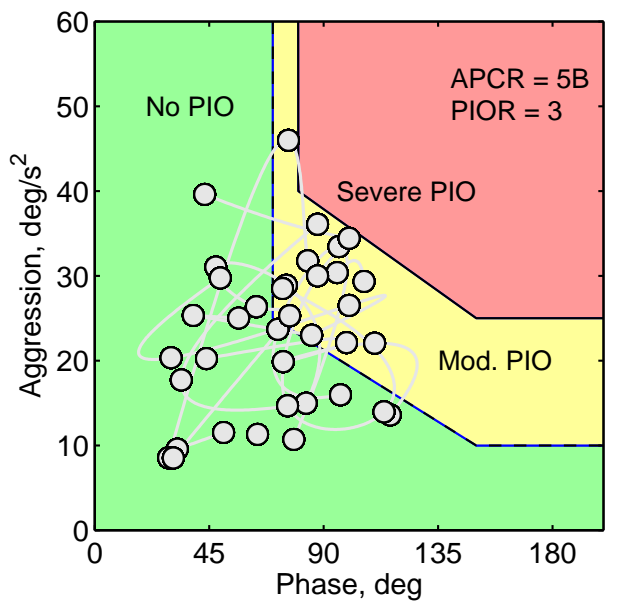

(e) PAC results - Case 5 - Pilot C

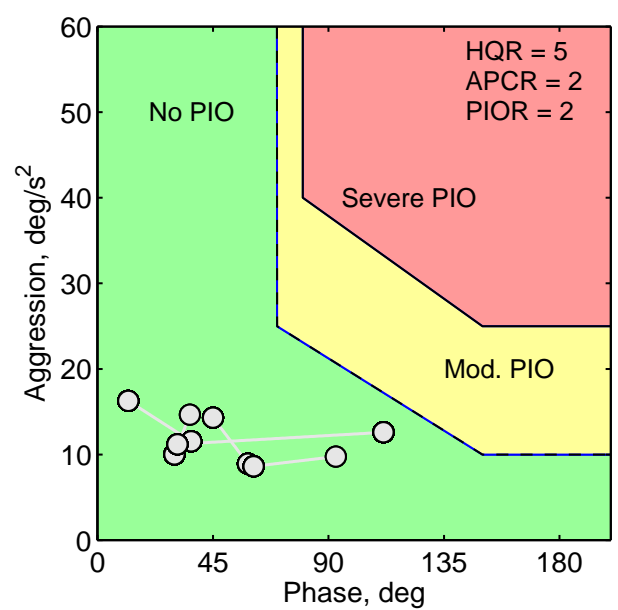

(b) PAC results - Case 2 - Pilot A

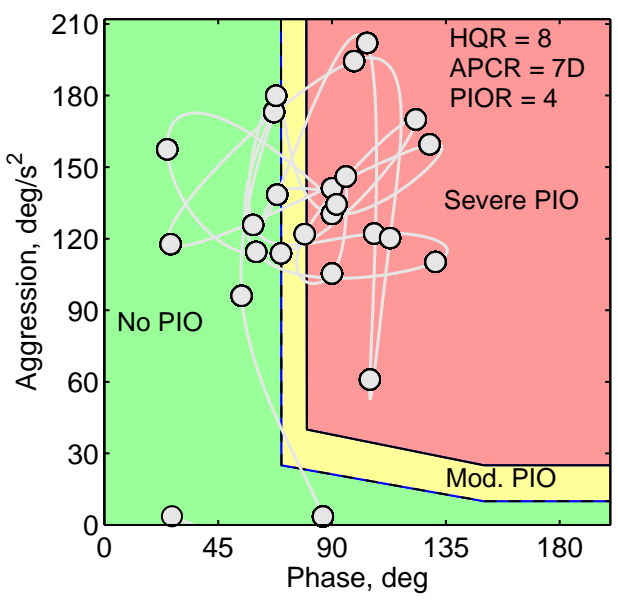

(d) PAC results - Case 4 - Pilot A

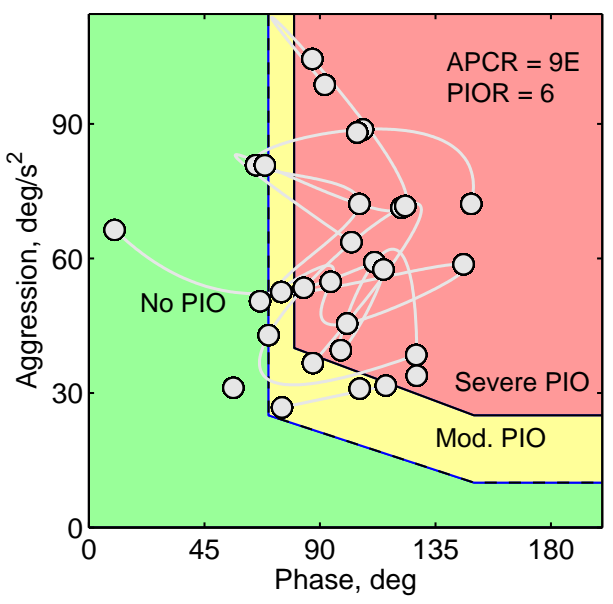

(f) PAC results - Case 6 - Pilot B

Figure 13: Example PAC results for cases investigated 


\section{F. Power Frequency Analysis}

Further analysis of cases was conducted using power frequency (PF) metrics, proposed in Ref. 29. The metric is an extension to cutoff frequency metrics. Time varying cutoff frequency is calculated using the power at a given time input. Therefore, the relative power with respect to the power at other times during the manoeuvre is lost. For time-invariant tasks, this is perhaps not a problem (i.e. sum-of-sines tracking, etc). However, for HQ tasks, where task requirements usually change throughout completion of the MTE, this can lead to high cutoff frequency in periods of low activity. To alleviate this issue, the metric shown in Eqn. 5 has been proposed,

$$
\omega_{G}\left(t_{i}\right)=\omega_{c o}\left(t_{i}\right) \max _{\omega}\left(S\left(t_{i}\right)\right)
$$

where $\omega_{G}$ is the $\mathrm{PF}, \omega_{c o}$ is the time varying cuttoff frequency, and $S\left(t_{i}\right)$ is the power spectral density of the signal used to calculate the PF. This modulates the cutoff frequency by the maximum power. When using the power frequency metric, in periods of low activity (and hence low power), $\omega_{G}$ will be low. The metric may also be used to find a single value for the complete test case. PF metrics have been recently applied to rotorcraft test cases. ${ }^{30}$ Figure 14 shows the maximum PF of the longitudinal force input with respect to pilot and test case. The mean $\mathrm{PF}$ and range obtained for each test case is also shown.

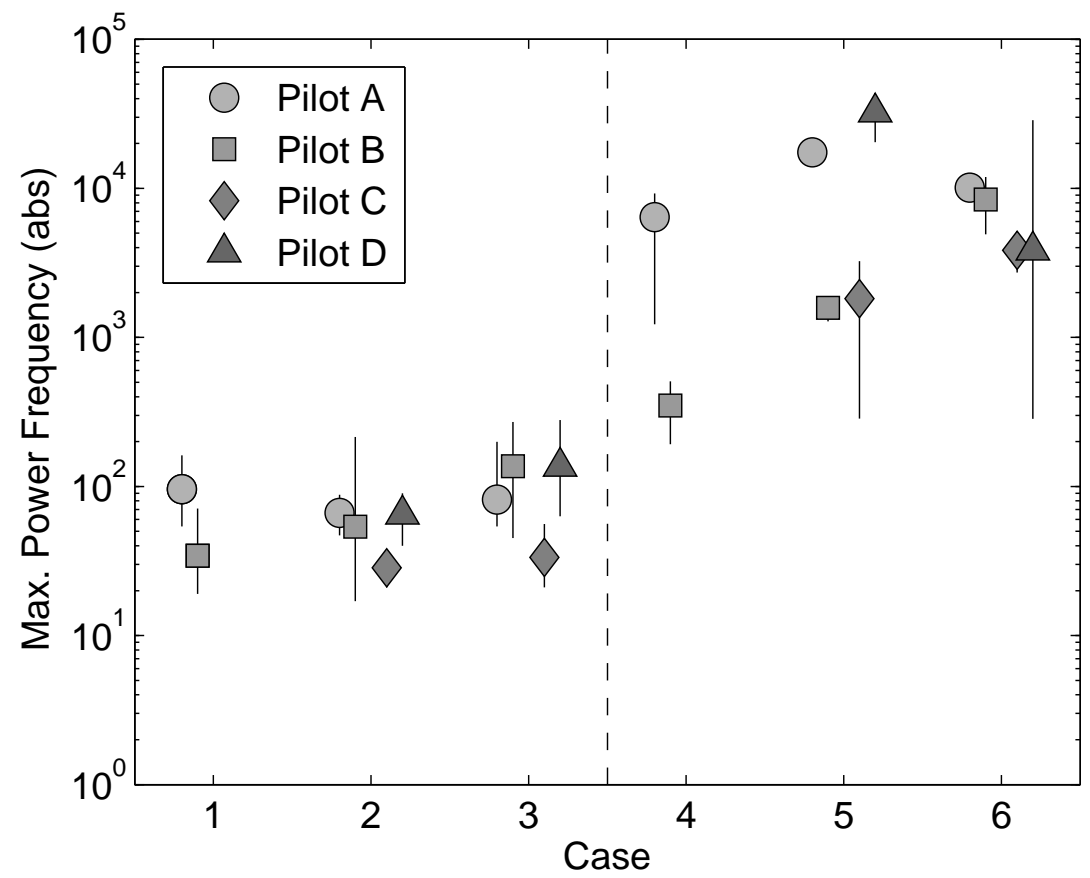

Figure 14: Maximum power frequency of longitudinal cyclic input force

The PF metric reflects PIOR and APCR awarded during the investigation. For the cases without isometric failure, only small changes are found in the maximum PF. With the additional time delay (Case 3), a small increase in PF is shown. For the failure cases, the PF significantly increases for all pilots. Pilots $\mathrm{A}$ and $\mathrm{D}$ were found to have the highest maximum PF. This correlates well with the objective analysis of data, whereby these two pilots were the most aggressive and entered into the most extreme APC conditions. Case 5 and Case 6 were found to have the highest maximum PF, which correlates well to APC and PIO assessment. Regarding Case 4, there is a large difference between Pilot A and Pilot B maximum PF. This supports the different IFES ratings awarded by the pilots, and differences in PIOR/APC ratings for the case. From results shown, it appears that the PF metric may be used to isolate failure cases and/or APC/PIO cases. This is due to the visible difference between failure and non-failure cases. 


\section{Discussion}

Prior to the pilot-in-the-loop investigations, it was expected that A/RPCs may occur during completion of the MTE. The MTE was deliberately selected as it features high aggression and tight performance tolerances. However, the dramatic nature of the oscillations, and the dependency upon the isometric failure was not entirely expected.

It is clear from the investigations conducted in the current research that the failure mode leads to difficult control for the pilot. Biodynamically, when the inceptor is 'frozen' in position, the pilot has no proprioceptive feedback and must adapt to judge her/his force input. In normal operation, the pilot is regulating the position input. $\mathrm{S} /$ he is aware that, as you reach the control limits and margins, dangerous flight conditions will materialise. This however is not apparent during failure, as there is no direct feedback available to the pilot. Following sudden isometric failure of the control inceptor, all pilots applied excessive force to compensate. This was, for most cases, found to trigger dynamic PIOs. As the pilot force exerted was much larger than for normal operations, runaway limiters were reached, which caused PIOs. For a large portion of these oscillations, the pilot could not bring the vehicle under control without releasing the control inceptor and/or abandoning the task.

In the failed state, the inceptor force was limited to $\pm 100 \mathrm{~N}(\approx 22 \mathrm{lbs})$ of force, at which point saturation limiting occurs. The lack of proprioceptive feedback means that the pilot is constantly over-controlling the vehicle. In the case of these investigations, this was mostly observed in the pitch axis. In some cases, pilot force following failure was a factor of 10 larger than prior to failure.

The dynamics of the situation were very dependent upon the flight condition during failure. When failure occurred in the 'worst-case' condition (i.e. point of maximum attitude during deceleration), oscillations were generally uncontrollable (at least during the transient phase). As the flight condition stability increased, the severity of oscillations decreased. Moreover, when the failure was initiated during a stable hover, with no task requirements, only very minor oscillatory tendencies were observed. This reflects findings during flight test, where only minor oscillations were experienced.

Whilst not discussed in detail within this paper, the control position during failure was found to significantly contribute to the severity of PIOs. A number of pilots commented that the lateral position of the control had a significant effect on the required compensation in the axis. As a result, the divided attention, and cross-coupling of the vehicle, oscillations developed and grew in intensity.

One observation from this research is that it appears that PIO tendencies could be mitigated against through the use of analysis techniques presented. OLOP showed prior to the investigations that the vehicle was PIO prone with the runaway limiter configuration. When flying with isometric failure and limiting as used in this investigation, further redesigns of the control parameters should be completed to mitigate against the RPC tendencies. This would account for the aggressive pilot control/abnormal vehicle operation. Although this investigation has highlighted PIO potential when using isometric failure modes, within the ACT/FHS this is only within the EP command channel. As a result, the safety concept of the aircraft removes the potential for PIO during flight testing using this mode.

The results of this investigation indicate that a potentially novel method of converting force signals to FCC/FCS inputs is required during situations of isometric failure. Throughout this investigation, forcedisplacement characteristics were as similar as possible before and after failure. High sensitivity following failure, and larger achievable forces suggest that a redesign of the force-displacement characteristics for the failure condition is necessary. Furthermore, assistance to pilots during the recovery and transition phase may remove RPC potential, as a reduction in PIO severity was shown for failure cases during stable conditions.

\section{Conclusions}

The implications of isometric inceptor failure, or any mode switching, can lead to dramatic aircraft/rotorcraft pilot coupling (A/RPC) events if not considered. In this investigation, it was shown that for all cases where runaway limiters were present, pilot induced oscillations (PIO) were experienced following failure. Furthermore, no pilot experienced severe PIOs during the completion of the manoeuvre without isometric failure. The following are the key conclusions from this investigation.

- The Open-Loop Onset Point (OLOP) criteria has been successfully applied to a case with non-linear control parameters. The application of approximations of rate limiting elements, independent of control 
input amplitude, was found to result in Cat. II PIO predictions. The OLOP results correlated well with experiences during flight during isometric failure. Cat. II PIOs were not observed without failure.

- The Phase Aggression Criteria (PAC) has been applied to the case of isometric inceptor failure. To achieve this, PAC input signals were modified for force input rather than displacement. Boundaries identified in previous investigations were found to be suitable.

- The maximum power frequency metric was used to assess differences in pilot force input for both failure and non-failure cases. A clear difference in metrics was found, indicating that power frequency could in future be used to determine when isometric failure occurs.

- A combination of APC prediction tools were used during the investigation. The tools used successfully distinguished PIO prone and robust cases. Features identified can be used in future research when looking further into PIO classification.

PIO susceptibility should always be considered during modifications to the vehicle or the experimental set-up. A wealth of tools, some of which have been utilised in this paper, are available for both the prediction and in-flight (and in-simulation) evaluation of PIO tendencies. Consideration of the impact of failure modes should be made throughout test programs. Furthermore, the consideration of the 'worst-case', as shown in this research, should also be paramount. Although isometric failure may have acceptable failure characteristics during low-gain flying tasks, when closed-loop performance (high-gain MTE) is required, the characteristics may be unacceptable.

Ongoing research at DLR is investigating handling qualities (HQ) and safety critical issues concerning the use of active control system (AIS) sidesticks. The research presented in this paper will be used as motivation for improvements in control dynamics and subsequent HQs following AIS failures (both isometric and isotonic). Further research in this area will utilise tools employed during these efforts.

\section{Acknowledgements}

The authors wish to thank Mario Müllhäuser for his assistance during the preparation of isometric failure test campaigns. Further thanks is extended to all pilots who participated in the investigations.

\section{References}

\footnotetext{
${ }^{1}$ Anon., "Aeronautical Design Standard Performance Specification Handling Qualities Requirements for Military Rotorcraft," Tech. Rep. ADS-33E-PRF, United States Army Aviation and Missile Command Aviation Engineering Directorate, 2000.

${ }^{2}$ Taylor, A., Greenfield, A., and Sahasrabudhe, V., "The Development of Active Inceptor Systems and the Scope and Design Issues of Tactile Cueing Systems," Proceedings of the American Helicopter Society 69th Annual Forum, AHS International, Phoenix, Arizona, 2008.

${ }^{3}$ Muellhaeuser, M., "Tactile Cueing with Active Cyclic Stick for Helicopter Obstacle Avoidance," Proceedings of the German Aerospace Congress, Braunschweig, Germany, 2016.

${ }^{4}$ von Gruenhagen, W., Schoenenberg, T., and Lantzsch, R., "Handling Qualities Studies into the Interaction between Active Sidestick Parameters and Helicopter Response Types," Proceedings of the American Helicopter Society 69th Annual Forum, AHS International, Phoenix, USA, 2013.

${ }^{5}$ Anon., "Aviation Accident Data Summary: Accident Number DCA16FA199," Tech. rep., National Transportation Safety Board, 2018.

${ }^{6}$ Kaletka, J. and Butter, U., "FHS, the New Research Helicopter: Ready for Service," Proceedings of the 29th European Rotorcraft Forum, Friedrichshafen, Germany, 16-18 September 2003.

${ }^{7}$ Pavel, M. D., Jump, M., Vu, B. D., Masarati, P., Genaretti, M., Ionita, A., Zaicek, L., Smaili, H., Quaranta, G., Yilmaz, D., Jones, M., Serafini, J., and Malecki, J., "Adverse Rotorcraft Pilot Couplings - Past, Present and Future Challenges," Progress in Aerospace Sciences, Vol. 62, 2013, pp. 1-51.

${ }^{8}$ McRuer, D. T., Droste, C. S., Hansman, R. J., Hess, R. A., LeMaster, D. P., Matthews, S., McDonnell, J. D., McWha, J., Melvin, W. W., and Pew, R. W., Aviation Safety and Pilot Control: Understanding and Preventing Unfavorable Pilot-Vehicle Interactions, National Research Council, Washington, D.C., 19971997.

${ }^{9}$ Mitchell, D. G. and Klyde, D. H., "Identifying a PIO signature - New techniques applied to an old problem," 2006 Atmospheric Flight Mechanics Conference, August 21, 2006 - August 24, Vol. 2, American Institute of Aeronautics and Astronautics Inc, Keystone, CO, United states, 2006, pp. 992-1006.

${ }^{10}$ Muscarello, V., Masarati, P., Quaranta, G., Lu, L., Jump, M., and Jones, M., "Invesitgation of Adverse Aeroelastic Rotorcraft-Pilot Coupling Using Real-Time Simulation," Proceedings of the 69th American Helicopter Society Annual Forum, 2013.
} 
${ }^{11}$ Anon., "Final Report: Accident occurred to the AgustaWestland AW609 Aircraft Registration Mark N609AG," Tech. rep., Agenzia Nazionale Per La Sicurezza Del Volo (ANSV), 2017.

${ }^{12}$ Duda, H., Gerlach, T., and Advani, S., "Design of the DLR AVES Research Flight Simulator," AIAA Modeling and Simulation Technologies (MST) Conference, 19-22 August 2013.

${ }^{13}$ Jones, M. and Jump, M., "New Methods to Subjectively and Objectively Evaulate Adverse Pilot Coupling," Journal of the American Helicopter Society, Vol. 60, No. 1, 2015, pp. 1-13.

${ }^{14}$ Mitchell, D. G., Arencibia, A. J., and Munoz, S., "Real-Time Detection of Pilot-Induced Oscillations," AIAA Atmospheric Flight Mechanics Conference, Vol. 1, American Institute of Aeronautics and Astronautics Inc, Providence, RI, United states, 2004, pp. 1-12.

${ }^{15}$ Gray, W. R., "Handling Qualities Evaluation at the USAF Test Pilot School," AIAA Atmospheric Flight Mechanics Conference, USAF Test Pilot School, AIAA, Chicago, Illinois.

${ }^{16}$ McRuer, D. T., "Pilot-Induced Oscillations and Human Dynamic Behavior," Tech. Rep. NASA Contractor Report 4683 , NASA, 1995

${ }^{17}$ von Gruenhagen, W., Muellhaeuser, M., Hoefinger, M., and Lusardi, J. A., "In-flight Evaluation of Active Sidestick Parameters with Respect to Handling Qualities for Rate Command and Attitude Command Response Types," Proceedings of the American Helicopter Society Handling Qualities Specialist Meeting, Huntsville, USA, 2014.

${ }^{18}$ Lehmann, P., Jones, M., and Hoefinger, M., "Impact of Turbulence and Degraded Visual Environment on Pilot Workload," CEAS Aeronautical Journal, Vol. 8, No. 3, 2017, pp. 413-428.

${ }^{19}$ Hamers, M., Graser, H., Bickel, N., Doktorczyk, M., Kistler, H., and Lantzsch, R., "Extension of the ACT/FHS Experimental Flight Envelope," Proceedings of the American Helicopter Society 65th Annual Forum, AHS International, Grapevine, Texas, 2009.

${ }^{20}$ Duda, H., "Prediction of Pilot-in-the-loop Oscillations Due to Rate Saturation," Journal of Guidance, Control, and Dynamics, Vol. 20, No. 3, 1997, pp. 581-587.

${ }^{21}$ Pavel, M. D., Malecki, J., Vu, B. D., Masarati, P., Quaranta, G., Gennaretti, M., Jump, M., Smaili, H., Ionita, A., and Zaichik, L. E., "Aircraft and Rotorcraft Pilot Coupling: a survey of recent research activities within the European project ARISTOTEL," 3rd CEAS Air and Space Conference, October 24-28 2011.

${ }^{22}$ Wilmes, T. and Duda, H., "Investigation of electronic filters to prevent aircraft-pilot-coupling," Tech. Rep. IB 111-98/29, German Aerospace Centre, 19981998.

${ }^{23}$ Duda, H., "Flying Qualities criteria considering rate limiting; Fliegbarkeitskriterien bei begrenzter Stellgeschwindigkeit," Tech. rep., German Aerospace Center, 1997.

${ }^{24}$ Tischler, M. B., Ivler, C. M., Mansur, M. H., Cheung, K. K., Berger, T., and Berrios, M., "Handling-Qualities Optimization and Trade-offs in Rotorcraft Flight Control Design," RAeS Rotorcraft Handling-Qualities Conference, Aeroflightdynamics Directorate (AMRDEC), US Army Research, Development, and Engineering Command; University Affiliated Research Center (UARC), University of California, Santa Cruz, Royal Aeronautical Soc, Liverpool, UK, 2008.

${ }^{25}$ DiFranco, D. A., "Flight Investigation of Longtudinal Short Period Frequency Requirements and PIO Tendencies," Tech. Rep. AFFDL-TR-66-163, Cornell Aeronautical Lab, 1967.

${ }^{26}$ Weingarten, N. and Chalk, C. R., "In-flight Investigation of Large Airplane Flying Qualities for Approach and Landing," Tech. Rep. AFWAL-TR-81-3118, Calspan, 1981.

${ }^{27}$ Mitchell, D. G. and Klyde, D. H., "Testing for Pilot-Induced Oscillations," AIAA Atmospheric Flight Mechanics Conference, Vol. 1, American Institute of Aeronautics and Astronautics Inc, San Francisco, CA, United states, 2005, pp. 98-111.

${ }^{28}$ Hindson, W. S., Eshow, M. M., and Schroeder, J. A., "A Pilot Rating Scale for Evaluating Failure Transients in Electronic Flight Control Systems," Proceedings of the AIAA Atomspheric Flight Mechanics Conference, AIAA, Portland, Oregon, 1990.

${ }^{29}$ Lampton, A. and Klyde, D. H., "Power Frequency: A Metric for Analyzing Pilot-in-the-Loop Flying Tasks," Journal of Guidance, Control, and Dynamics, Vol. 35, No. 5, 2012, pp. 1526-1537.

${ }^{30}$ Tritschler, J., O'Connor, J., Holder, J., Klyde, D. H., and Lampton, A., "Interpreting Time Frequency Analyses of Pilot Control Activity in ADS-33E Mission Task Elements," 73rd AHS Annual Forum, AHS International, Fort Worth, TX, 2017. 